\title{
A RELATIONSHIP IN SPHERICAL HARMONICS
}

\author{
BY J. H. WEBB
}

It is known that a solid spherical harmonic about one set of axes can be expressed as a solid spherical harmonic with constant coefficients about a new set of axes whose origin is displaced from that of the first. A new relationship has been found for making this transformation and a proof is herein given.

Consider the two sets of cartesian coordinates $x, y, z$ and $x_{1}, y_{1}, z_{1}$, whose origins $O$ and $O_{1}$ are separated by a distance $h$. Let the two axes $x$ and $x_{1}$ be coincident, but let the positive sense of the two axes be opposite. Consider the point $S$ which has the two sets of polar coordinates defined by the equations

$$
\begin{aligned}
& x_{1}=\rho_{1} \cos \theta_{1}=\rho_{1} \mu_{1}, \quad x=\rho \cos \theta=\rho \mu, \\
& y_{1}=\rho_{1} \sin \theta_{1} \sin \phi_{1}, \quad y=\rho \sin \theta \sin \phi, \\
& z_{1}=\rho_{1} \sin \theta_{1} \cos \phi_{1}, \quad z=\rho \sin \theta \cos \phi .
\end{aligned}
$$

The transformation to be obtained may be expressed symbolically as follows:

$$
\sum_{m=0}^{\infty} \rho_{1}^{-(m+1)} Y_{m}\left(\mu_{1}, \phi_{1}\right)=\sum_{m=0}^{\infty} f_{m}(\rho) Y_{m}(\mu, \phi),
$$

in which $Y_{m}$ signifies the usual spherical harmonic. Such a transformation has been given previously by $\mathrm{B}$. Datta.* The new relationship which has been found to give the transformation (1) and which appears to have been unnoticed before is

$$
\frac{P_{m^{n}}\left(\mu_{1}\right)}{\rho_{1}^{m+1}}=a_{(m-n)}\left(1-\mu^{2}\right)^{n / 2} \frac{\partial^{m-n}}{\partial h^{m-n}}\left(\frac{1}{h^{n}} \frac{\partial^{n}}{\partial \mu^{n}} \frac{1}{\rho_{1}}\right),
$$

in which $a_{(m-n)}$ designates the term $(-1)^{m-n} /(m-n) !$. A proof of this relationship follows.

Relative to the partial differentiation on the right side of (2), $\rho_{1}$ is to be expressed in terms of $\rho, \mu$ and $h$. At certain stages of the calculation it will be convenient to express $\rho_{1}$ by means of the equation

* Tôhoku Mathematical Journal, vol. 15 (1919), p. 166. 


$$
\frac{1}{\rho_{1}}=\frac{1}{h} \sum_{k=0}^{\infty}\left(\frac{\rho}{h}\right)^{k} P_{k}(\mu),
$$

and at others by the equation

$$
\frac{1}{\rho_{1}}=\left[\rho^{2}-2 \rho h \mu+h^{2}\right]^{-1 / 2}=R^{-1} .
$$

Differentiating $\rho_{1}^{-1}$ as given by equation (4), with respect to $\mu$, $n$ times and dividing by $(\rho h)^{n}$, gives

$$
(\rho h)^{-n} \frac{\partial^{n} \rho_{1}^{-1}}{\partial \mu^{n}}=1 \cdot 3 \cdots(2 n-1) R^{-(2 n+1)},
$$

and this may be simplified so as to read

$$
h^{-n} \frac{\partial^{n} \rho_{1}^{-1}}{\partial \mu^{n}}=c(n) \frac{\rho^{n}}{\rho_{1}^{2 n+1}},
$$

in which $c(n)$ designates the term $(2 n) ! /\left(2^{n} n !\right)$. Furthermore by the law of sines, (see Fig. 1),

$$
\left(1-\mu^{2}\right)^{n / 2}=\left(1-\mu_{1}^{2}\right)^{n / 2}\left(\frac{\rho_{1}}{\rho}\right)^{n} .
$$

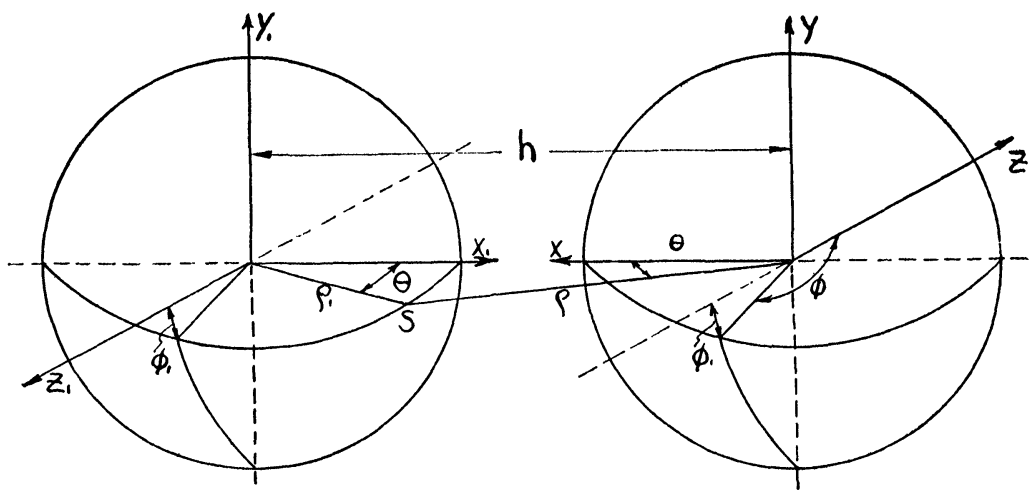

FIG. 1

Substituting the above value in the right side of equation (2) and replacing the left side by its value from the defining equation 


$$
\frac{P_{m}^{n}\left(\mu_{1}\right)}{\rho_{1}^{m+1}}=\frac{\left(1-\mu_{1}^{2}\right)^{n / 2}}{\rho_{1}^{m+1}} \frac{\partial^{n} P_{m}\left(\mu_{1}\right)}{\partial \mu_{1}^{n}}
$$

we obtain in place of equation (2) the equation

$$
\left(2^{\prime}\right) \frac{\left(1-\mu_{1}^{2}\right)^{n / 2}}{\rho_{1}^{m+1}} \frac{\partial^{n} P_{m}\left(\mu_{1}\right)}{\partial \mu_{1}^{n}}=a_{(m-n)}\left(1-\mu_{1}^{2}\right)^{n / 2} c(n) \frac{\partial^{m-n}}{\partial h^{m-n}} \rho_{1}^{-(n+1)},
$$

and this may be written as

$$
\frac{1}{\rho_{1}^{m+n+1}} \frac{\partial^{n} P_{m}\left(\mu_{1}\right)}{\partial \mu_{1}^{n}}=a_{(m-n)} c(n) \frac{\partial^{m-n}}{\partial h^{m-n}} \rho_{1}^{-(2 n+1)} .
$$

It has been shown* that the following equation is true:

$$
\text { (8) } \begin{aligned}
& \frac{\partial^{n} P_{m}\left(\mu_{1}\right)}{\partial \mu_{1}{ }^{n}}=\frac{c(m)}{(m-n) !}\left[\mu_{1}^{m-n}-\frac{(m-n)(m-n-1)}{2[2 n+2(m-n)-1]^{m-n-2}} \mu_{1}^{m-n}(m-n-1)(m-n-2)(m-n-3)\right. \\
+ & \left.\frac{(m-n)(m-n)-1][2 n+2(m-n)-3]}{2 \cdot 4[2 n+2(m-n)-n-4}-\cdots\right] .
\end{aligned}
$$

Therefore to establish equation (2), it is only necessary to show that the equation

$$
(-1)^{m-n} c(n) \frac{\partial^{m-n}}{\partial h^{m-n}} \rho_{1}^{-(2 n+1)}=\frac{1}{\rho_{1}^{m+n+1}} \frac{\partial^{n} P_{m}\left(\mu_{1}\right)}{\partial \mu_{1}^{n}}
$$

is true. This can be shown by an induction argument applied to the parameter $(m-n)$. First note that the proposed formula is true for $(m-n)=1$. Then $m=n+1$ and the proposed formula reads

$$
c(n) \frac{\partial}{\partial h} R^{-(2 n+1)}=c(n+1) \frac{\mu_{1}}{\rho_{1}^{2} n+2},
$$

or

$$
R^{-(2 n+3)}(h-\rho \mu)=\mu_{1} \rho_{1}^{-(2 n+2)} .
$$

Replacing $(h-\rho \mu)$ by $\rho_{1} \mu_{1}$, we easily verify that this equation is an identity. Therefore, let the validity of the proposed equation, for index $(m-n)$, be assumed and show that it holds when $(m-n)$ is replaced by $(m-n+1)$. That is, let the following equation be assumed:

\footnotetext{
* Byerly, Fourier Series and Spherical Harmonics, 1893, p. 197.
} 


$$
\begin{aligned}
& (-1)^{m-n} \frac{\partial^{m-n} \rho_{1}^{-(2 n+1)}}{\partial h^{m-n}}=(2 n+1)(2 n+3) \cdots \\
& \cdot\{2 n+2(m-n)-1\}\left[\frac{\left(\rho_{1} \mu_{1}\right)^{m-n}}{\rho_{1}^{[2 n+2(m-n)+1]}}\right. \\
& -\frac{(m-n)(m-n-1)}{2[2 n+2(m-n)-1]} \frac{\left(\rho_{1} \mu_{1}\right)^{m-n-2}}{\rho_{1}^{[2 n+2(m-n)-1]}} \\
& +\frac{(m-n)(m-n-1)(m-n-2)(m-n-3)}{2 \cdot 4[2 n+2(m-n)-1][2 n+2(m-n)-3]} \\
& \quad \frac{\left(\rho_{1} \mu_{1}\right)^{m-n-4}}{\left.\rho_{1}^{[2 n+2(m-n)-3]}-\cdots\right]} .
\end{aligned}
$$

Equation (11) differs formally from equation (9), whose validity is to be established, in that the coefficient of the square bracket on the right side has been modified in form. The purpose of this modification is to bring into explicit evidence the parameter $(m-n)$ to which the induction process applies. Replace $\rho_{1} \mu_{1}$ in (11) by its value, $(h-\rho \mu)$, differentiate once with respect to $h$ and replace $(h-\rho \mu)$ in the resulting equation by $\left(\rho_{1} \mu_{1}\right)$. The resulting equation is

$$
\begin{aligned}
(-1)^{m-n+1} \frac{\partial^{m-n+1}}{\partial h^{m-n+1}} \rho_{1}^{-(2 n+1)}=(2 n+1)(2 n+3) \cdots \\
\quad \cdot\{2 n+2(m-n)+1\}\left[\mu_{1}^{m-n+1}\right. \\
-\frac{(m-n+1)(m-n)}{2[2 n+2(m-n)+1]} \mu_{1}^{m-n-1} \\
\left.+\frac{(m-n+1)(m-n)(m-n-1)(m-n-2)}{2 \cdot 4[2 n+2(m-n)+1][2 n+2(m-n)-1]} \mu_{1}^{m-n-3}-\cdots\right] .
\end{aligned}
$$

By comparing equation (12) with (11), it is seen that wherever $(m-n)$ occurs in (11), $(m-n+1)$ occurs in (12), thus proving the validity of equation (11).

If now $\rho_{1}^{-1}$ be replaced in equation (2) by its value as given by equation (3), the result is 


$$
\frac{P_{m}^{n}\left(\mu_{1}\right)}{\rho_{1}^{m+1}}=a_{(m-n)}\left(1-\mu^{2}\right)^{n / 2} \frac{\partial^{m-n}}{\partial h^{m-n}} \frac{1}{h^{n}} \frac{\partial^{n}}{\partial \mu^{n}} \sum_{k=0}^{\infty} \frac{\rho^{k}}{h^{k+1}} P_{k}(\mu)
$$

Differentiating with respect to $\mu$ and $h$, we obtain

(14) $\frac{P_{m}^{n}\left(\mu_{1}\right)}{\rho_{1}^{m+1}}=\sum_{k=n}^{\infty} \frac{(m+k) !}{(m-n) !(n+k) !} \frac{\rho^{k}}{h^{m+k+1}} P_{k}^{n}(\mu)=\sum_{k=n}^{\infty} \alpha_{k n m}$.

For the special case $n=0$, (14) reduces to the form*

$$
\frac{P_{m}\left(\mu_{1}\right)}{\rho_{1}{ }^{m+1}}=\sum_{k=0}^{\infty} \frac{(m+k) !}{m ! k !} \frac{\rho^{k}}{h^{m+k+1}} P_{k}(\mu)=\sum_{k=0}^{\infty} \alpha_{k 0 m} . \dagger
$$

The formulas (14) and (15) furnish a means of obtaining the desired transformation (1). In fact the left member of equation (1), written in full, is

$$
\begin{aligned}
\sum_{m=0}^{\infty} A_{0 m} \sum_{k=0}^{\infty} \alpha_{k 0 m} & +\sum_{n=1}^{m}\left[A_{n m} \cos n\left(180^{\circ}-\phi\right)\right. \\
& \left.+B_{n m} \sin n\left(180^{\circ}-\phi\right)\right] \sum_{k=n}^{\infty} \alpha_{k n m} .
\end{aligned}
$$

By an interchange of the indices $m$ and $k$, and by noting that $\cos n(180-\phi)=(-1)^{n} \cos n \phi$ and $\sin n\left(180^{\circ}-\phi\right)=\sin n \phi$, (16) can be thrown into the desired form,

$$
\sum_{m=0}^{\infty}\left[\sum_{k=0}^{\infty} A_{0 k} \alpha_{m 0 k}+\sum_{n=1}^{m} \sum_{k=n}^{\infty}\left(A_{n k} \cos n \phi+B_{n k} \sin n \phi\right) \alpha_{m n k}\right]
$$

and it can be shown that such a rearrangement of terms in the triple series is legitimate providing the $A_{n k}$ and the $B_{n k}$ are bounded. It is to be noted that $A_{n k}=(-1)^{n} A_{n k}$.

In conclusion, I should like to express my thanks to Professor Warren Weaver of the University of Wisconsin for his helpfulness and interest in the above work.

Williams College

* See Whittaker and Watson, Modern Analysis, 4th edition, p. 400, ex. 7.

$\uparrow$ See B. Datta, loc. cit., p. 170. 